\title{
Article \\ Flowing Refractometer for Feed Water State Control in the Second Loop of Nuclear Reactor
}

\author{
Vadim Davydov ${ }^{1,2, *}$, Irena Gureeva ${ }^{1}$, Roman Davydov ${ }^{3}\left(\mathbb{D}\right.$ and Valentin Dudkin ${ }^{4}(\mathbb{D}$ \\ 1 Institute of Electronics and Telecommunications, Peter the Great St. Petersburg Polytechnic University, \\ 195251 St. Petersburg, Russia; irena-gureeva@mail.ru \\ 2 All Russian Research Institute of Phytopathology, 143050 Moscow, Russia \\ 3 Institute of Physics and Mechanics, Peter the Great St. Petersburg Polytechnic University, \\ 195251 St. Petersburg, Russia; davydovroman@outlook.com \\ 4 Department of Photonics and Communication Lines, The Bonch-Bruevich Saint Petersburg State University of \\ Telecommunication, 193232 St. Petersburg, Russia; vidoodkin@mail.ru \\ * Correspondence: davydov_vadim66@mail.ru
}

Citation: Davydov, V.; Gureeva, I.; Davydov, R.; Dudkin, V. Flowing Refractometer for Feed Water State Control in the Second Loop of Nuclear Reactor. Energies 2022, 15, 457. https://doi.org/10.3390/ en15020457

Academic Editors: Hiroshi Sekimoto and Sung Joong Kim

Received: 29 November 2021

Accepted: 4 January 2022

Published: 10 January 2022

Publisher's Note: MDPI stays neutral with regard to jurisdictional claims in published maps and institutional affiliations.

Copyright: (C) 2022 by the authors. Licensee MDPI, Basel, Switzerland. This article is an open access article distributed under the terms and conditions of the Creative Commons Attribution (CC BY) license (https:// creativecommons.org/licenses/by/ $4.0 /)$.

\begin{abstract}
The necessity to control the feed water state in the second loop of a nuclear power plant nuclear reactor is justified. The different methods of the state control of flowing water in the pipeline are reviewed. It has been established that controlling the feed water state should not result in irreversible changes in its chemical composition and physical structure. A change in the composition or structure of feed water leads to a change in its heat capacity. The heat transfer deteriorates, the production of electrical energy in the installation decreases, and the additional release of heat into the atmosphere increases. This process also occurs during the heat capacity changes for other reasons. The method for controlling the feed water heat capacity by measuring the value of the refractive index $n$ is developed. The design of a flow-through refractometer based on the total internal reflection for control of the feed water state in the stream is made. The dependence of the heat capacity change of feed water from the refractive index is established. The results of research on different types of water are presented.
\end{abstract}

Keywords: nuclear power plant; nuclear reactor; feed water; pipeline; refractometer; medium refractive index; heat capacity; heat exchange

\section{Introduction}

At present, nuclear energy provides a significant proportion of humanity's needs for electrical energy and, in some cases, thermal energy [1-7]. This direction of energy is constantly evolving since it has an undeniable advantage over other types of electrical and thermal power plants [2,3,5-9]. Regardless of weather conditions, a nuclear power plant (NPP) allows you to provide the required power level at any time of the day [4-7]. It is essential for large industrial areas. In addition, at a nuclear power plant, it is possible to regulate the generated power level within $20 \%$ of the nominal power level. More than $30 \%$ of regulation is included in new models of nuclear power plants. This will significantly reduce the cost of generating electricity. At NPPs, increased attention is paid to reducing electricity costs and increasing environmental safety [8-12].

One of the ways to reduce costs in the production of electrical energy is to increase the efficiency of heat exchange in the second loop of the reactor between the coolant and feed water. The efficiency of heat exchange (transfer of thermal energy from the coolant to the feed water) is determined by several parameters (interaction time, which depends on the flow rate of media in pipelines) and the specific heat capacities of these media $[13,14]$. In the case of a change, for example, a decrease in the specific heat capacity of the feed water will result in changes to the amount of heat transferred. This will decrease heat transfer efficiency and can be compensated by reducing the consumption of feed water 
$q$ (the time of interaction with the coolant will increase). The feed water must be heated in the pipeline to a temperature of $493 \mathrm{~K}$ (or more, depending on the model of the steam generator) [13-15].

During the operation of a nuclear power plant, various chemical processes occur in the secondary circuit's pipelines. These processes alter the composition and structure of the nutrient water. In addition, when the feed water in the steam generator is converted into steam and then into the water, various impurities can enter it, changing the feed water composition. Therefore, at the NPP, measures are taken to purify feed water. These measures are expensive since they are associated with a decrease in the volume of electricity produced.

At present, at NPPs, the heat capacity of feed water $C$ is monitored based on pressure and temperature measurements. For this, various devices are used, which are explicitly made for nuclear power plants (for example, in the Russian Federation-AIR-30, in the USA-the $3200 \mathrm{G}$ series). These devices provide pressure measurements ranging from $0.1 \mathrm{MPa}$ to $100 \mathrm{MPa}$ up to a temperature of $600 \mathrm{~K}$ with a measurement error of $0.3-0.5 \%$ (depending on the temperature regime). The interval between instrument verifications is three years (this is not enough, since the instrument must be removed from the pipeline during validation, the technological cycle in this pipeline is interrupted). In addition, under the new operating conditions of the nuclear power plant, this measurement error is insufficient to ensure the optimal heat transfer regime.

It should be noted that the control of the feed water condition with this device cannot be carried out. The control of the feed water condition is carried out using sampling at certain intervals. Further, these samples are sent to a stationary laboratory for research. The time interval between sampling and obtaining data on the state of the feed water can be several hours. This results in wasted decision time and electrical power. It is necessary to automatically monitor the state of the current feed water in real-time.

Therefore, it is advisable to carry them out only when necessary. As such, the purpose of our work is to develop a method for monitoring the state of feed water in real-time to ensure an optimal heat transfer regime and its practical implementation.

\section{Methodology for Monitoring the State of Feed Water and Its Practical Implementation}

Currently, monitoring the state of the current environment in real-time in the pipeline is a complicated task [7,16-21]. This is especially the case at high pressures, temperatures, and flow rates in the pipeline. In this case, to ensure the long-term operation of the measuring device required at a nuclear power plant, it is necessary to use non-contact methods of monitoring the current environment. This is an optical method based on refraction and a nuclear magnetic method based on the phenomenon of nuclear magnetic resonance (NMR) [7,21-25]. The optical (refractometric) method has several advantages over the nuclear magnetic method. Refractive index measurements are independent of the fluid flow rate, and there are no restrictions on the pipeline's diameter [26-29]. Therefore, we have developed the following technique. The state of the feed water determines the value of the refractive index $n$ (any change in feed water composition leads to a change in $n$ ). The value of the heat capacity $C$ of the feed water is also determined by its composition. Any changes in the composition lead to a change in $C$. At a given temperature $T$ in the feed water, a specific value of $n$ corresponds to a specific value of $C$. It is possible to plot the $C(n)$ dependence for the feed water at a given temperature. There will be a different temperature of the feed water's different dependence $C(n)$. Further, $n$ and $T$ are measured in the current flow of feed water. Using the previously obtained calibration curves $C(n)$ in a stationary laboratory on devices with a measurement error of $10^{-4}$ or less, the value of $C$ is determined. This value is used to adjust the flow rate $q$ of the feed water to ensure the optimal heat exchange mode.

To implement this technique, we have developed a design of a flow-through refractometer using the phenomenon of total internal reflection [26-34]. The use of this phenomenon in a refractometer allows the measurement error of $\mathrm{n}$ from 0.00005 to 0.0002 in dependence on temperature regime. The basis of any refractometer is its optical part, which 
we developed to measure the refractive index of feed water at high temperatures [29-38]. Figure 1 shows a block diagram of the optical part of the refractometer and the propagation of laser beams in it.

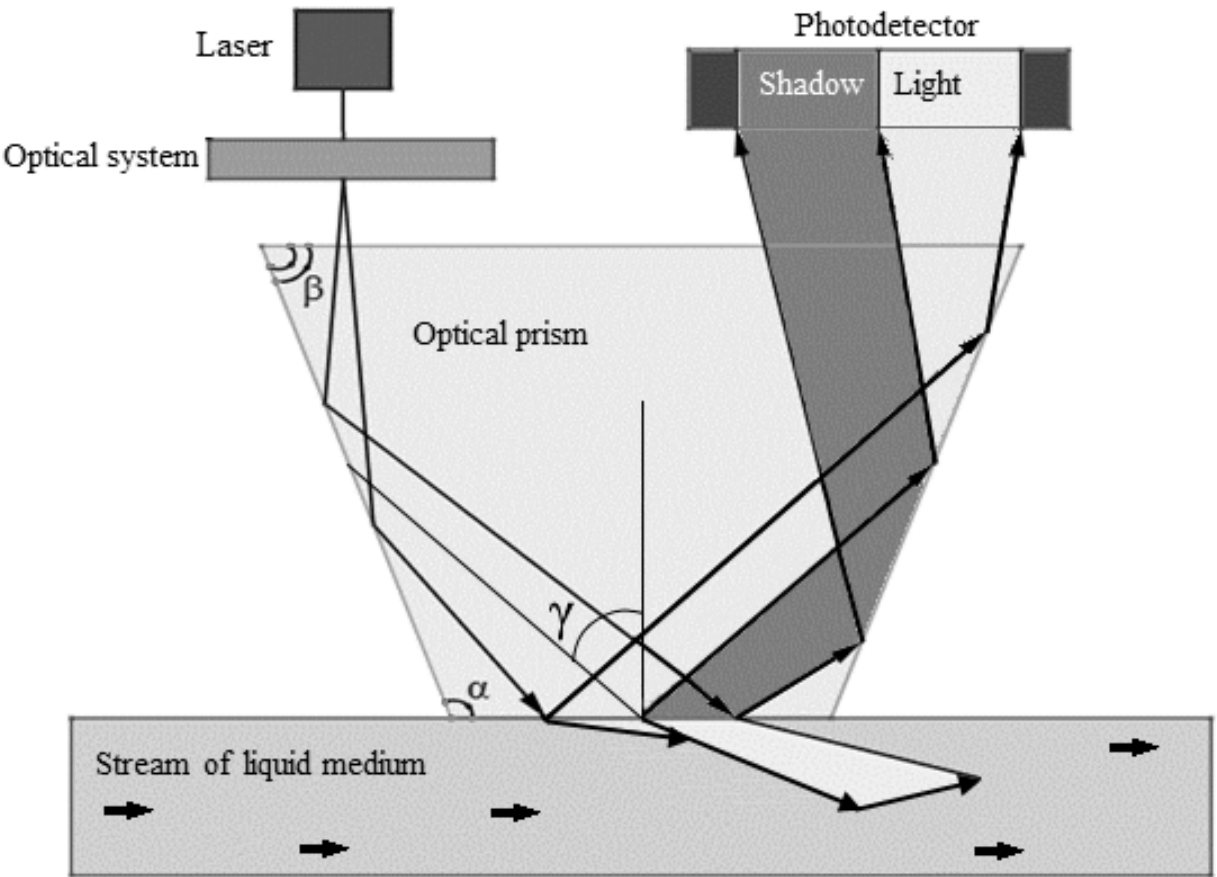

Figure 1. Structural diagram of the optical part of a refractometer.

The design of the optical part of the refractometer is a prism in the form of a trapezoid with an angle at the bottom base $\beta=60$ degrees and at the upper base $\alpha=120$ degrees. The prism is made of leucosapphire. The leucosapphire has high chemical resistance (density $3950-4100 \mathrm{~kg} / \mathrm{m}^{3}$ ) and a high melting point (2093-2123 K). The prism angles were calculated to provide the maximum contrast of the light/shadow boundary recorded in the photodetector and the range change maximum in time registration of light/shadow border displacement in case of a grand change of $n$. The refractive index $n$ in the developed design of the refractometer is measured as follows. A preliminary calculation of the propagation of the maximum laser radiation in the prism is performed (the prism angles are given). The relationship between the position of the light-shadow boundary on the photodetector (photodiode ruler) and the angle of total internal reflection of the laser beam $\gamma$ (Figure 1) is determined. The value of the angle $\gamma$ is determined by Equation (1).

$$
\gamma=\arcsin \left(\frac{n_{f}}{n_{p}}\right)
$$

where $n_{p}$ is the refractive index of the material from which the prism is made (leucosapphire), and $n_{f}$ is the refractive index of the feed water.

The angle $\gamma$ corresponds to a certain maximum position on the photodetector. The maximum of the laser radiation coincides with the position of the light-shadow boundary recorded on the photodetector. The position of the light-shadow boundary determines $\gamma$. Next, using Equation (1), $n_{f}$ is determined.

For this, in the developed design of the refractometer, the laser is installed perpendicular to the prism's base. The rays reach the interface between the prism and the liquid medium at different angles. An additional optical system is used that extends the beam of laser radiation.

Part of the rays, the angle of incidence of which is more $\gamma$, completely reflect off the inner surface of the prism and, emerging from it, form the light part of the image on the photodetector. Other rays, the angle of incidence of which is less than $\gamma$, partially refract 
and pass into the feed water, where they partially reflect and form the dark part of the image on the photodetector.

The position of the interface between light and shadow depends on the ratio between the refractive indices of the prism material and medium and their temperatures. In the case of composition changes in the feed water, its refractive index $n$ changes, and the position of the light-shadow boundary recorded by the photodetector is shifted.

Figure 2 presents a structural diagram of a refractometer developed by us with the trapezoidal prism for state control of flowing feed water in the pipeline.

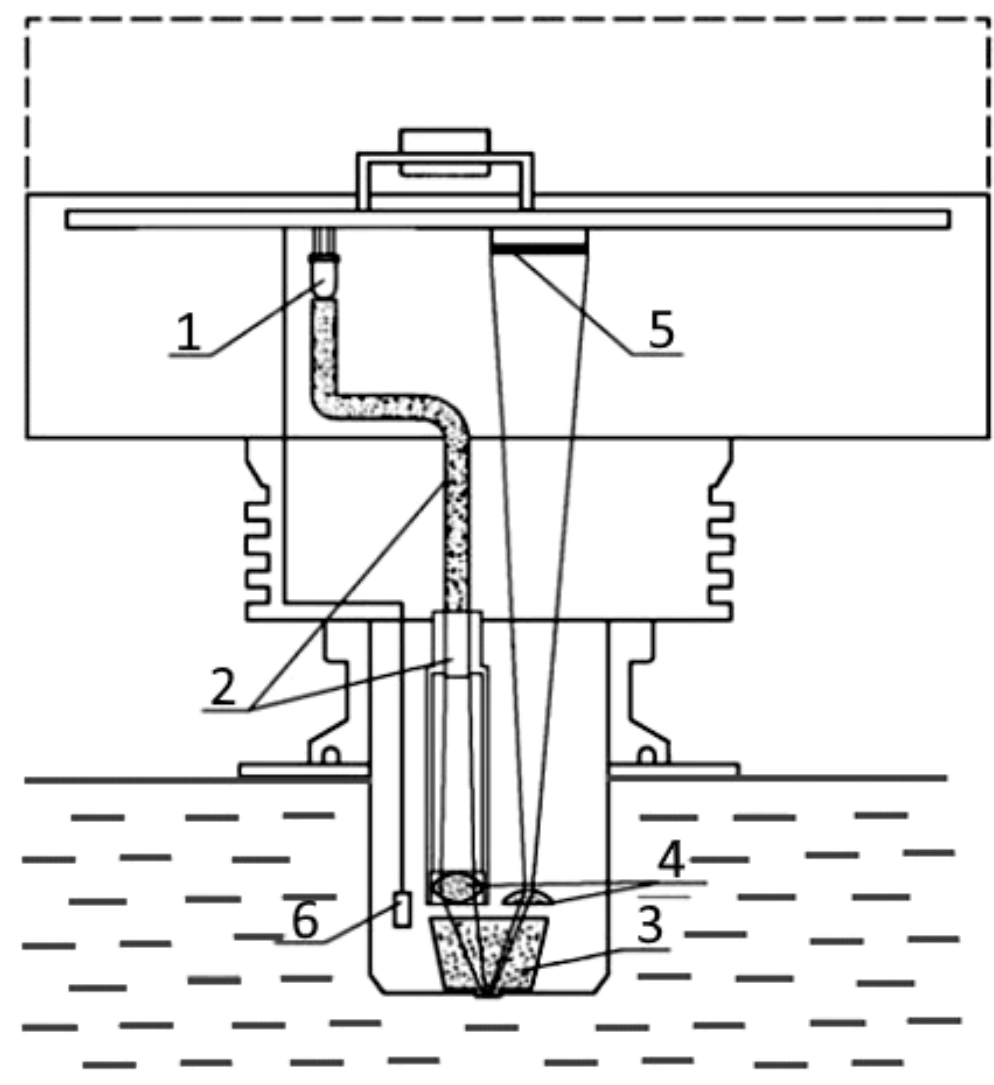

Figure 2. Structural diagram of an industrial refractometer: (1) radiation source, (2) fiber optic bundle, (3) optical prism, (4) lens, (5) CCD array, (6) temperature sensor.

The optical system design developed for the refractometer allows it to be immersed in the pipeline only until it comes into contact with the flowing liquid. It makes it possible not to introduce additional hydraulic resistance into the pipeline during measurements, which affects the heat transfer coefficient between the coolant and the feed water.

\section{Results and Discussion}

Figure 3 shows, as an example, the dependences of the change in the refractive index $n$ of various flowing media through the pipeline on the temperature $T$, measured using our design of a refractometer on an experimental stand. The pressure of the flowing medium in the pipeline is $2.4 \mathrm{MPa}$. 


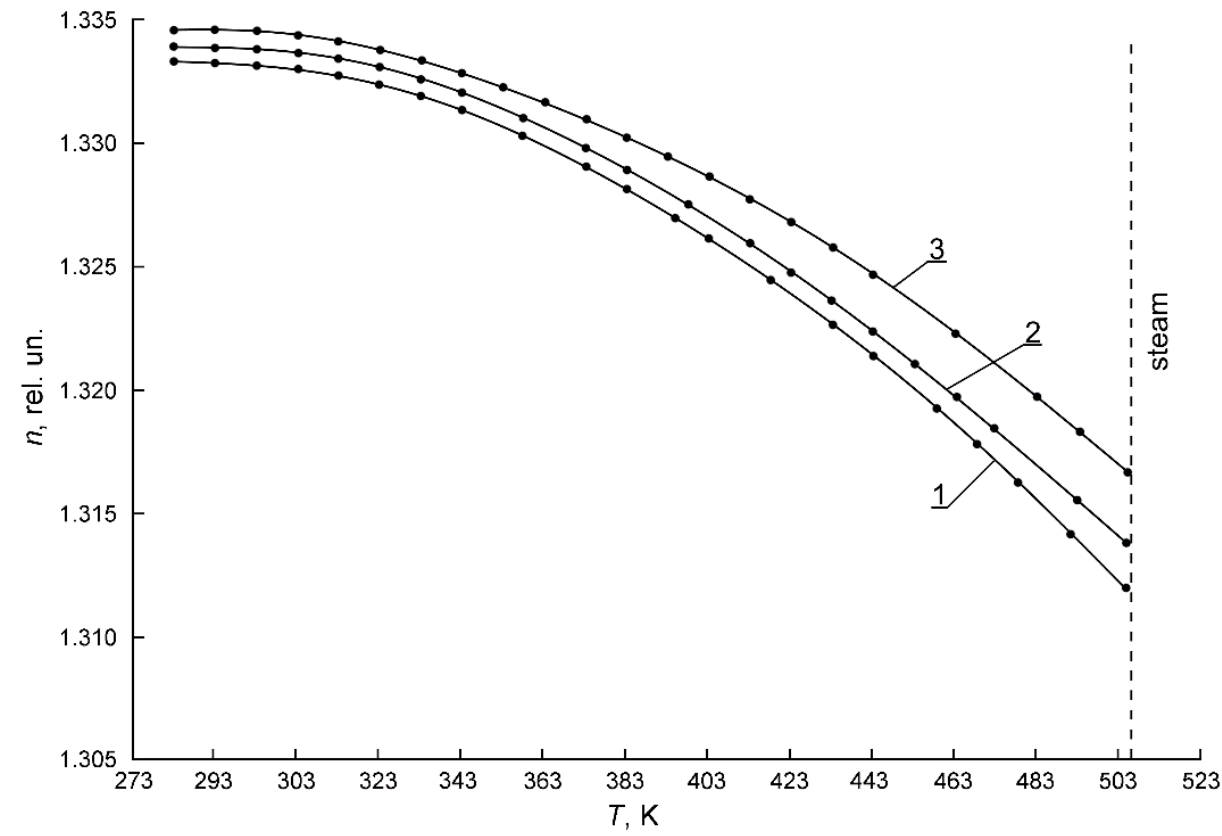

Figure 3. Dependence of the refractive index $n$ on the temperature T. Graphs (1-3) correspond to the following media: distilled water, feed water and tap water.

The obtained results of measuring the refractive indices coincided with the measurement error with the data obtained by us on a stationary Abbe refractometer (NAR-2T UH) with a temperature control unit. The measurement error of $n$ is 0.0002 for the stationary Abbe refractometer (NAR-2T UH). As an example, for temperature $T=343.1 \mathrm{~K}$, Table 1 shows the measurements comparison results made with two refractometers.

Table 1. Refractive indices $\mathrm{n}$ of feed water are measured by different types of refractometers.

\begin{tabular}{ccc}
\hline & \multicolumn{2}{c}{ Refractometer Type } \\
\cline { 2 - 3 } $\mathbf{N}^{\mathbf{0}}$ & The Developed Flowing Refractometer & Abbe Refractometer (NAR-2T UH) \\
\hline 1 & $1.3317 \pm 0.0003$ & $1.3316 \pm 0.0002$ \\
2 & $1.3318 \pm 0.0003$ & $1.3315 \pm 0.0002$ \\
3 & $1.3317 \pm 0.0003$ & $1.3315 \pm 0.0002$ \\
4 & $1.3316 \pm 0.0003$ & $1.3315 \pm 0.0002$ \\
5 & $1.3318 \pm 0.0003$ & $1.3316 \pm 0.0002$ \\
6 & $1.3317 \pm 0.0003$ & $1.3317 \pm 0.0002$ \\
7 & $1.3317 \pm 0.0003$ & $1.3317 \pm 0.0002$ \\
8 & $1.3316 \pm 0.0003$ & $1.3317 \pm 0.0002$ \\
9 & $1.3316 \pm 0.0003$ & $1.3315 \pm 0.0002$ \\
10 & $1.3318 \pm 0.0003$ & $1.3316 \pm 0.0002$ \\
11 & $1.3318 \pm 0.0003$ & $1.3316 \pm 0.0002$ \\
12 & $1.3316 \pm 0.0003$ & $1.3315 \pm 0.0002$ \\
13 & $1.3317 \pm 0.0003$ & $1.3315 \pm 0.0002$ \\
14 & $1.3317 \pm 0.0003$ & $1.3317 \pm 0.0002$ \\
15 & $1.3317 \pm 0.0003$ & $1.3317 \pm 0.0002$ \\
16 & $1.3316 \pm 0.0003$ & $1.3316 \pm 0.0002$ \\
17 & $1.3316 \pm 0.0003$ & $1.3316 \pm 0.0002$ \\
18 & $1.3318 \pm 0.0003$ & $1.3317 \pm 0.0002$ \\
19 & $1.3317 \pm 0.0003$ & $1.3315 \pm 0.0002$ \\
20 & $1.3318 \pm 0.0003$ & $1.3315 \pm 0.0002$ \\
\hline
\end{tabular}

The data analysis presented in Table 1 showed that the results of measurements of $n$ for all numbers (No.) coincide within the measurement error. The developed device is distinguished by the high accuracy and reliability of measurements. 
Figure 4 shows the dependence of the change in the specific heat $C$ on the temperature $T$.

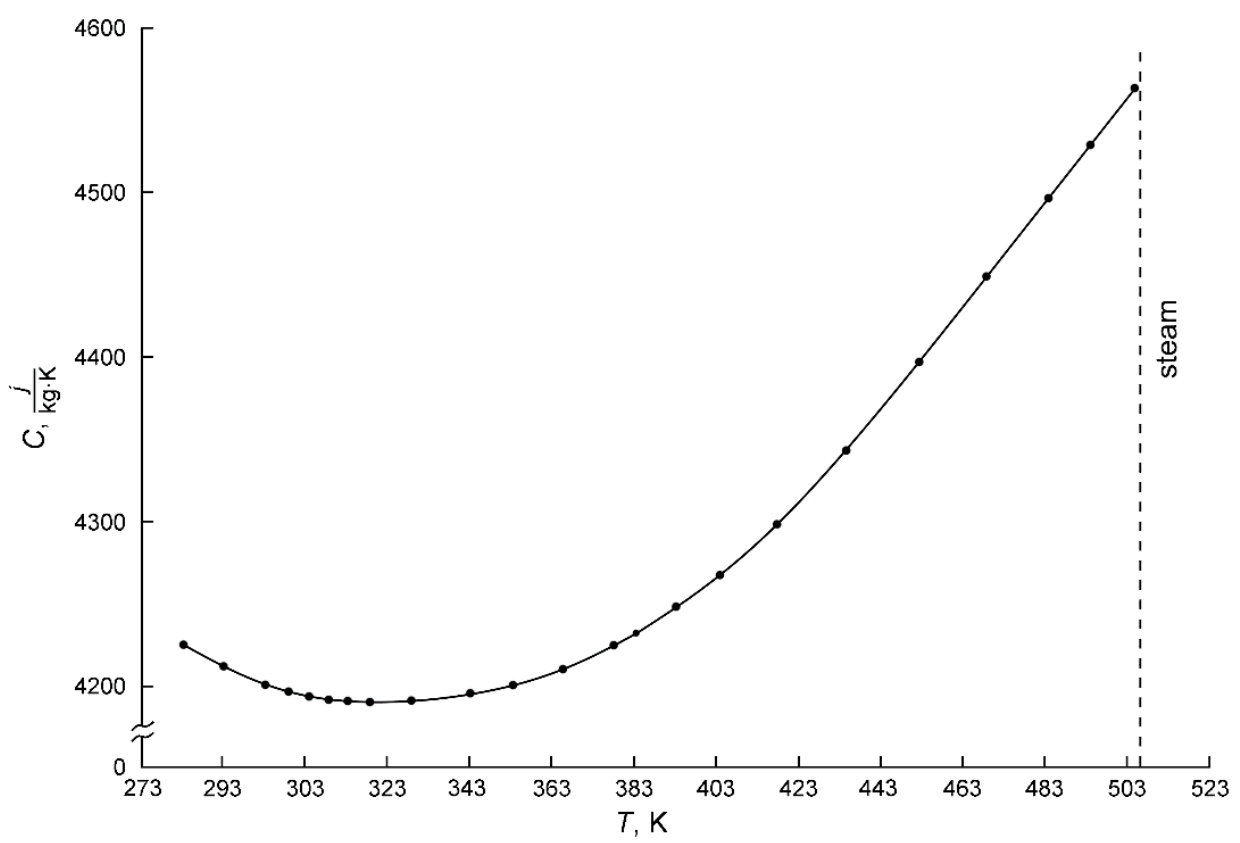

Figure 4. The change in the specific heat capacity $C$ of the feed water on the temperature $T$.

It is possible to construct a calibration curve for the change in the specific heat capacity of feed water $C$ from a change in the refractive index $n$ comparing Figures 3 and 4 . Figure 5 shows this dependence.

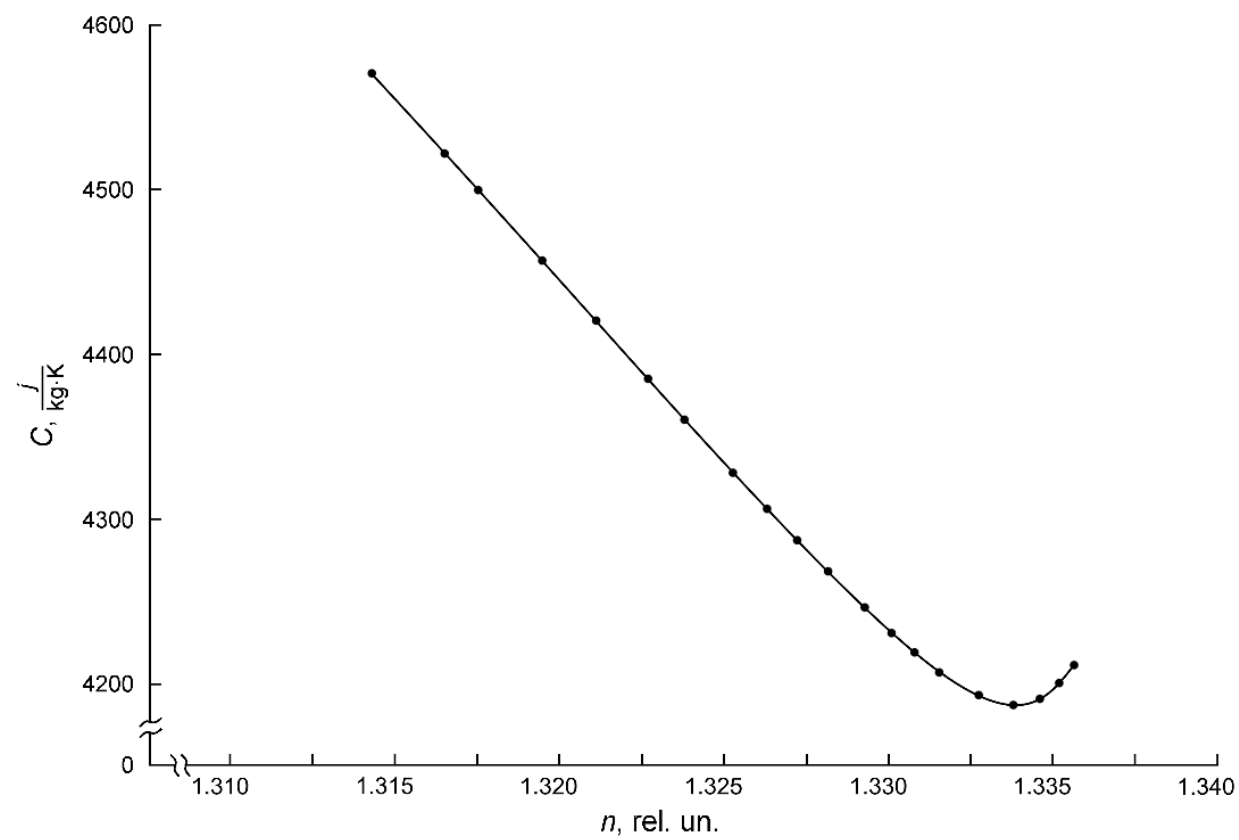

Figure 5. The ratio between the value of the specific heat capacity $C$ of the feed water and the value of its refractive index $n$.

Based on the obtained result, it is possible to note the close to linear character of the $C(n)$ dependence in the range of $T$ change from 350 to $504 \mathrm{~K}$; that is, the operating range of the feed water temperature change in most models of steam generators at nuclear power plants. The technological capabilities of the experimental stand (pressure limitations up to 
2.4 $\mathrm{MPa}$ ) did not allow us to heat the feed water above the temperature of $507 \mathrm{~K}$ (at a given $T$, it turned into steam). At several nuclear power plants, feed water at a higher pressure is heated to a temperature of $600 \mathrm{~K}$. The nature of the $C(n)$ dependence in the region of these temperatures will need to be investigated additionally to use the method we have developed to control $C$.

In addition, the refractometer developed by us makes it possible to establish the reasons for the sharp change in the heat capacity $C$ in the pipeline. Figure 6 shows the results of studying the refractive index of feed water from the temperature in the presence of various impurities.

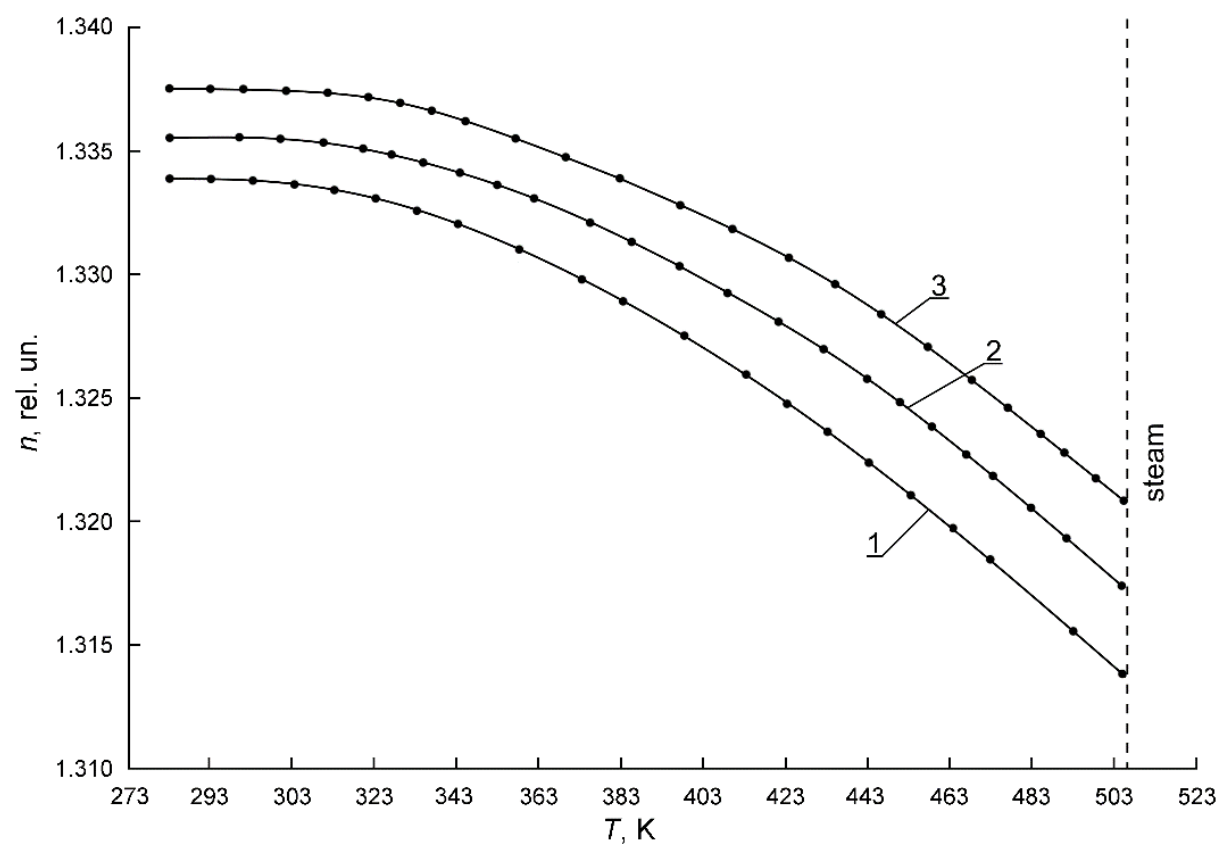

Figure 6. The dependence of the refractive index $n$ of the feed water on the temperature $T$. Graphs (1-3) correspond to the following: absence of impurities in water, presence of iron oxides and carbon steel in water, presence of technical oils in water.

Analysis of the results obtained shows a sharp change in the value of the refractive index $n$ of the feed water at a constant temperature T. Considering that water is an incompressible liquid, such an abrupt change in $n$ cannot be caused by an abrupt change in pressure in the pipeline. In addition, our studies have shown that a change in pressure by $1 \mathrm{MPa}$ in feed water corresponds to a change in the refractive index $n$ by 0.0005 . Therefore, this change in $n$ is unambiguously associated with the appearance of impurities. By analyzing the dependence of the change in $n$ on $T$, it is possible to determine the cause of the appearance of impurities.

To ensure the reliability of the transmission of information about the measured refractive index of the feed water from a refractometer installed on the pipeline of the steam generator system, a fiber-optic communication line (FOCL) is used. Modern designs of FOCL are not sensitive to electromagnetic interference and vibrations. They can be used at air temperatures up to $573 \mathrm{~K}$ and above.

\section{Conclusions}

Our results showed that measuring the refractive index and temperature of the flowing feed water in the pipeline in real-time makes it possible to control the change in its specific heat capacity $C$. It allows us to ensure the optimal heat transfer coefficient (Nusselt criterion) between the coolant and the feed water. In this case, the electrical energy generation will be maximum without overloading the steam generator set. The nuclear power plant will operate normally. 
Preliminary estimates have shown that the use of our methodology for monitoring the state of feed water (with subsequent adjustment of its flow rate in the pipeline when $C$ changes) can increase the service life of the steam generator and reduce the number of preventive cleanings of feed water during the operation of a nuclear power plant by at least two to three times. These conclusions were drawn based on the first data on the results of prototypes of refractometers on an experimental bench with a steam generator.

It should also be noted that the refractometer developed by us allows us to control the quality of feed water (the presence of impurities in it) and establish the cause of its appearance.

Another advantage of the developed device is its uninterrupted service life compared to the pressure meter, which is now used at nuclear power plants. The pressure meter verification period is three years. It is due to the design of the instrument and the formation of calcium deposits in it. These deposits sharply increase the device error up to $5-7 \%$. The service life of these meters is from 10 to 12 years (then replacement). All these procedures are associated with the interruption of the technological cycle. The refractometer developed by us does not require verification (after preliminary calibration before installation) for 15 years (further, it must be checked due to the degradation of electronics when working in a high-temperature area). Automatic cleaning of the optical element, which is in contact with the feed water, allows the refractometer to operate continuously for 15 years. This is another indisputable advantage of the method developed by us compared to those currently used at nuclear power plants.

It should also be noted that the refractometer developed by us makes it possible to measure the refractive index $n_{f}$ (heat capacity $C$ ) in the case of stationary feed water in the pipeline (the main central pump is turned off). The water temperature in the pipeline is continuously measured, but there is almost no pressure $P$ in the pipeline. In this case, it is extremely difficult to determine $C$ (using $P$ ) (there will be a huge measurement error). This is another additional function for monitoring the status of the feed water.

The authors plan to continue their refraction studies in the current environment to reduce the measurement error in the developed design of a flow-through refractometer. The phenomenon of the Goos-Hanchen shift makes a significant contribution to its formation (about 0.0001). It must be studied in detail at various temperature conditions to develop methods for compensating this phenomenon.

Author Contributions: Conceptualization, R.D. and V.D. (Vadim Davydov); methodology, V.D. (Vadim Davydov); software, R.D.; validation, I.G., V.D. (Vadim Davydov) and R.D.; formal analysis, I.G and V.D. (Vadim Davydov); investigation, R.D.; resources, V.D. (Valentin Dudkin) and I.G.; data curation, I.G.; writing - original draft preparation, V.D. (Vadim Davydov); writing-review and editing, R.D.; visualization, R.D.; supervision, V.D. (Valentin Dudkin); project administration, V.D. (Vadim Davydov); funding acquisition, V.D. (Vadim Davydov). All authors have read and agreed to the published version of the manuscript.

Funding: The research is partially funded by the Ministry of Science and Higher Education of the Russian Federation under the strategic academic leadership program 'Priority 2030' (Agreement 075-15-2021-1333 dated 30 September 2021).

Institutional Review Board Statement: Not applicable.

Informed Consent Statement: Not applicable.

Conflicts of Interest: The authors declare no conflict of interest.

\section{References}

1. Agafonova, N.D.; Egorov, M.Y.; Sergeev, V.V.; Gotovskii, M.A.; Kruglikov, P.A.; Lebedev, M.E.; Sudakov, A.V.; Fedorovich, E.D.; Fokin, B.S. Heat-and-Mass Transfer Intensification in Saturated-Steam Generators in NPP with VVER as a Means for Increasing Efficiency and Reliability. At. Energy 2018, 123, 154-158. [CrossRef]

2. Gulevich, A.V.; Dekusar, V.M.; Chebeskov, A.N.; Kuchinov, V.P.; Voloshin, N.P. Possibility of Fast-Reactor Exportation Under an International Nuclear Non-Proliferation Regime. At. Energy 2020, 127, 192-195. [CrossRef] 
3. Ashurko, Y.M.; Gulevich, A.V.; Klinov, D.A.; Vasil'ev, B.A.; Vasyaev, A.V.; Marova, E.V.; Shepelev, S.F. Gen-IV Reactor Systems Criteria Implementation in BN-1200. At. Energy 2019, 125, 351-358. [CrossRef]

4. Dong, Z.; Liu, M.; Jiang, D.; Huang, X.; Zhang, Y.; Zhang, Z. Automatic Generation Control of Nuclear Heating Reactor Power Plants. Energies 2018, 11, 2782. [CrossRef]

5. Lee, K.-H.; Kim, M.-G.; Lee, J.I.; Lee, P.-S. Recent Advances in Ocean Nuclear Power Plants. Energies 2015, 8, 11470-11492. [CrossRef]

6. Wu, G.; Ju, P.; Song, X.; Xie, C.; Zhong, W. Interaction and Coordination among Nuclear Power Plants, Power Grids and Their Protection Systems. Energies 2016, 9, 306. [CrossRef]

7. Davydov, V.V.; Myazin, N.S.; Kiryukhin, A.V. Nuclear-Magnetic Flowmeter-Relaxometers for Monitoring Coolant and Feed water Flow and Status in Npp. At. Energy 2020, 127, 274-279. [CrossRef]

8. Davydov, R.; Antonov, V.; Makeev, S.; Batov, Y.; Dudkin, V.; Myazin, N. New high-speed system for controlling the parameters of a nuclear reactor in a nuclear power plant. E3S Web Conf. 2019, 140, 02001. [CrossRef]

9. Krapivtsev, V.G.; Solonin, V.I. Model Studies of Interloop Coolant Mixing in VVER-1000 in-Reactor Pressure Channel. At. Energy 2019, 125, 307-313. [CrossRef]

10. Elokhin, A.P. Unconventional Methods of Radiological Environmental Monitoring and Earthquake Prediction. At. Energy 2015, 117, 206-215. [CrossRef]

11. Ksenofontov, A.I.; Elokhin, A.P.; Alalem, E.A. Application of simulation modelling approaches for analyzing $\gamma$-radiation characteristics of a plume induced by a Nuclear Accident at NPP. Loon 2019, 487, 012009. [CrossRef]

12. Fadeenko, V.; Fadeenko, I.; Nikolaev, D. Remote environmental monitoring in the area of a nuclear power plant. IOP Conf. Ser. Earth Environ. Sci. 2019, 390, 012022. [CrossRef]

13. Sorokin, A.P.; Kuzina, Y.A. Physical Modeling of Hydrodynamics and Heat Transfer in Liquid-Metal Cooled Fast Reactors. At. Energy 2020, 128, 277-286. [CrossRef]

14. Filimonov, P.E.; Semchenkov, Y.M.; Malyshev, V.V.; Dolgopolov, N.Y.; Povarov, V.P.; Gusev, I.N. VVER-1200 Tests in No. 6 Unit of the Novovoronezh NPP During Operation in a Daily Load Schedule. At. Energy 2020, 129, 143-148. [CrossRef]

15. Abramov, L.V.; Baklanov, A.V.; Bakmetiev, A.M.; Bylov, I.A.; Vasyuchenkov, A.A.; Gusev, D.O.; Kiselev, V.V. OKBM Afrikantov Experience in Developing Methods and Computer Codes for Reliability Analysis and Probabilistic Safety Analysis of Nuclear Installations. At. Energy 2020, 129, 103-108. [CrossRef]

16. Firth, J.; Ladouceur, F.; Brodzeli, Z.; Wyres, M.; Silvestri, L. A novel optical telemetry system applied to flowmeter networks. Flow Meas. Instrum. 2016, 48, 15-19. [CrossRef]

17. Looney, R.; Priede, J. Concept of a next-generation electromagnetic phase-shift flowmeter for liquid metals. Flow Meas. Instrum. 2019, 65, 128-135. [CrossRef]

18. Novikova, O.V.; Erastov, A.E.; Livshits, S.A. Features of evaluating the efficiency indicators of the electric power enterprise. E3S Web Conf. 2019, 124, 05057. [CrossRef]

19. Medeiros, K.A.R.; Barbosa, C.R.H.; d'Almeida, J.R.M.; Ribeiro, A.S.; de Paula, I.B. Flowmeter based on a piezoelectric PVDF tube. Meas. J. Int. Meas. Confed. 2019, 138, 368-378. [CrossRef]

20. Marusina, M.Y.; Bazarov, B.A.; Galaidin, P.A.; Marusin, M.P.; Silaev, A.A.; Zakemovskaya, E.Y.; Mustafaev, Y.N. Design of a Gradient System for a Multiphase Flowmeter. Meas. Tech. 2014, 57, 580-586. [CrossRef]

21. Davydov, V.V.; Dudkin, V.I.; Karseev, A.Y.; Vologdin, V.A. Special Features in Application of Nuclear Magnetic Spectroscopy to Study Flows of Liquid Media. J. Appl. Spectrosc. 2016, 82, 1013-1019. [CrossRef]

22. Karabegov, M.A. The dynamic characteristics of automatic analyzers. Meas. Tech. 2013, 55, 1301-1310. [CrossRef]

23. Karabegov, M.A. Automatic differential prism refractometer for monitoring process liquids. Meas. Tech. 2007, 50, 619-628. [CrossRef]

24. Mishchenko, Y.V. Method of refractometric control of liquids in processing plants. Meas. Tech. 2007, 50, 1274-1281. [CrossRef]

25. Mishchenko, Y.V. Fiber-optic interferometric refractometer for deep-water studies of sea water. Meas. Tech. 2006, 49, 1210-1217. [CrossRef]

26. Shur, V.L.; Naidenov, A.S.; Lukin, A.Y.; Leibengardt, G.I. A liquid autocollimation refractometer. Meas. Tech. 2006, 49, 815-819. [CrossRef]

27. Davydov, V.V.; Grebenikova, N.M.; Smirnov, K.Y. An Optical Method of Monitoring the State of Flowing Media with Low Transparency That Contain Large Inclusions. Meas. Tech. 2019, 62, 519-526. [CrossRef]

28. Meeten, G.H.; North, A.N. Refractive index measurement of absorbing and turbid fluids by reflection near the critical angle. Meas. Sci. Technol. 1995, 6, 214-221. [CrossRef]

29. Contrerastello, H.; Márquezislas, R.; Vázquezestrada, O.; Sánchezpérez, C.; Garcíavalenzuela, A. Understanding the performance of Abbe-type refractometers with optically absorbing fluids. Meas. Sci. Technol. 2014, 25, 075201. [CrossRef]

30. Leibengardt, G.I.; Naidenov, A.S.; Shur, V.L. A liquid laser interference refractometer for measuring the concentration of solutions. Meas. Tech. 2004, 47, 1211-1216. [CrossRef]

31. Chen, J.; Guo, W.; Xia, M.; Li, W.; Yang, K. In situ measurement of seawater salinity with an optical refractometer based on total internal reflection method. Opt. Express 2018, 26, 25510-25523. [CrossRef] [PubMed]

32. Morales-Luna, G.; Garcia-Valenzuela, A. Viability and fundamental limits of critical-angle refractometry of turbid colloids. Meas. Sci. Technol. 2017, 28, 125203. [CrossRef] 
33. Niskanen, I.; Räty, J.; Peiponen, K.-E. On the Immersion Liquid Evaporation Method Based on the Dynamic Sweep of Magnitude of the Refractive Index of a Binary Liquid Mixture: A Case Study on Determining Mineral Particle Light Dispersion. Appl. Spectrosc. 2017, 71, 1586-1592. [CrossRef]

34. Morales-Luna, G.; Herrera-Domínguez, M.; Pisano, E.; Balderas-Elizalde, A.; Hernandez-Aranda, R.I.; Ornelas-Soto, N. Plasmonic biosensor based on an effective medium theory as a simple tool to predict and analyze refractive index changes. Opt. Laser Technol. 2020, 131, 106332. [CrossRef]

35. Morales-Luna, G.; García-Valenzuela, A.; Barrera, R.G. Optical Coherent Reflection from a Confined Colloidal Film: Modeling and Experiment. J. Phys. Chem. B 2018, 122, 8570-8581. [CrossRef]

36. Bhardwaj, V.K.; Maini, S. Compact and self-aligned fluid refractometer based on the Doppler-induced self-mixing effect. Appl. Opt. 2020, 59, 3064-3072. [CrossRef]

37. Luo, Y.; Guo, W.; Yu, L.; Xia, M.; Li, W.; Yang, K. High-precision algorithms for critical angle refractive index measurement. Appl. Opt. 2019, 58, 6057-6062. [CrossRef]

38. Guo, W.; Li, R.; Yu, L.; Chen, J.; Xia, M.; Li, W.; Yang, K. Self-referenced technology for refractive index measurement under mechanical vibration and temperature fluctuation. Appl. Opt. 2019, 58, 1862-1867. [CrossRef] [PubMed] 